\title{
NEW EDITOR FOR BJHS
}

\author{
We are very pleased indeed to report that
}

\section{DR JANET BROWNE}

is to be the new editor, responsible for issues from 1994 onwards, and taking up office in July 1993.

Her address is:

Wellcome Institute for the History of Medicine 183 Euston Road, London NW1 2BE Tel. 071-611-8657 Fax 071-611-8545 